\title{
Wavelet Image Denoising Based on The New Threshold Function
}

\author{
Tang Hui \\ Department of Communication Engineering \\ Kunming University of Science and Technology \\ KunMing , China \\ Email: thweibo@163.com
}

\section{Chen Lin}

Department of Communication Engineering Kunming University of Science and Technology KunMing, China

Email:_sealinya@foxmail.com

\author{
Liu Zengli \\ Department of Communication Engineering \\ Kunming University of Science and Technology \\ KunMing, China \\ Email: liuzengli@hotmail.com
}

Chen Zaiyu

Department of Communication Engineering

Kunming University of Science and Technology

KunMing, China

Email: chenzaiyu-1987@163.com

\begin{abstract}
A new threshold function was proposed to overcome that hard threshold function is not continuous, soft threshold function has constant deviation and derivative discontinuity defects. It will be applied to using different thresholds denoising method with different decomposition level based on the D.J global threshold. Experimental results shows that the denoising result of new threshold function is superior to the traditional soft and hard threshold function in minimum mean square error (MSE) and peak signal to noise ratio (PSNR).
\end{abstract}

Keywords-component; wavelet transform;image denoising; hard threshold; soft threshold

\section{INTRODUCTION}

Usually there will be various noise in the process of image acquisition and transmission. In order to improve the image quality, we must ensure that the denoised image maintains s many important signal characteristics as possible. Image denoising are paid more and more attention of scholars based on the theory of wavelet because wavelet transform has good local time-frequency, multi-scale and multi-resolution characteristics.

In 1995, Donoho and Johnstone proposed the concept of wavelet threshold denoising based on binary wavelet transform[1,2,3], i.e.the VisuShrink image denoising method. Wavelet threshold denoising has been widely used in many fields because it has the advantages of fast calculation speed, wide adaptability as well as being able to get the best estimation that any other linear estimates is not able to reach. However, the discontinuous of hard threshold function cause oscillation in signal reconstruction, the denoised image has the Pseudo-Gibbs phenomenon and other visual distortion, the differential coefficient of soft threshold function is not continuous and exists a constant deviation between the estimates of wavelet coefficients and the real wavelet coefficients as well as denoised image occurs edge blur, these disadvantages limits its further application. In 2001, Donoho improved the threshold algorithm[4], L.K.shark et al. gave the optimalizing threshold denoising method[5], but there is always a constant deviation between reconstructed signal and real signal. The reference[6] presents adaptive threshold denoising algorithm,but the setting of algorithm parameter is more complex.

In this paper, according to the different image noise level adaptive changes the contractile parameters of the new threshold function, we propose a new threshold method. Using different threshold denoising with the different decomposition level based on VisuShrink image denoising method proposed by Donoho. We demonstrate that our proposed method outperforms the traditional ones in terms of MSE and PSNR. Simulation results are also given to show the efficacy of our proposed method. The rest of the paper is organized as follows. Section 2 explains some basic concepts used in denoising with wavelet threshold. Section 3 describes our proposed denoising method. Experimental results and analysis are given in section 4. Finally, our concluding remarks are given in section 5 .

\section{DENOISING WITH WAVELET THRESHOLD}

Consider an original image signal of $f(i, j)$ size $\mathrm{M}^{*} \mathrm{~N}$ and noisy signal. Add the Gaussian noise to original image signal in order to get the noisy signal $g_{i, j}$, i.e.,

$$
g(i, j)=f(i, j)+n(i, j)
$$

The wavelet coefficients of the image signal is composed of two parts because the wavelet transform is a linear transformation, $W_{g}=W_{f}+W_{n}, W_{f}$ is the original image wavelet coefficients, $W_{n}$ is the noise wavelet coefficients, $W_{g}$ is the noisy image wavelet coefficients. The image wavelet coefficients of the signal is greater than the noise wavelet coefficients after wavelet decomposition, we can find a suitable value $T$ as a threshold based on wavelet 
threshold denoising method proposed by Donoho , i.e.the D.J global threshold.

Using the threshold value $T$ processing the wavelet coefficients, get the estimated wavelet coefficients, i.e.,

a)Hard threshold function

$$
\hat{W}_{i, j}=\left\{\begin{array}{cc}
W_{i, j} & \left|W_{i, j}\right| \geq T \\
0 & \left|W_{i, j}\right|<T
\end{array}\right.
$$

b)Soft threshold function

$$
\hat{W}_{i, j}=\left\{\begin{array}{cl}
\operatorname{sgn}\left(W_{i, j}\right)\left(\left|W_{i, j}\right|-T\right) & \left|W_{i, j}\right| \geq T \\
0 & \left|W_{i, j}\right|<T
\end{array}\right.
$$

Where $W_{i, j}$ is the high frequency coefficients of wavelet decomposition, when $W_{i, j}$ is less than the threshold value $T$, this is mainly caused by noise, discarded and replaced by zero, When $W_{i, j}$ is greater than the threshold value $T, W_{i, j}$ is mainly caused by the image signal, this part retained(hard threshold function) or contracted to zero according to a fixed value (soft threshold method), and then take inverse-wavelet transform to get the denoised image $\hat{f}(i, j)$.

In their landmark paper, Donoho and Johnstone have discussed a simple but powerful wavelet-based denoising scheme called VisuShrink, it uses the universal threshold value $\mathrm{T}$ which is proportional to the standard deviation of the noise, is defined as:

$$
T=\sigma \sqrt{2 \log M}
$$

Where $M$ is the total number of image pixels, $\sigma$ is the noise standard deviation, estimating through the $H_{1}$ subband of the wavelet transform[7,8,9], which is defined as:

$$
\hat{\sigma}=\operatorname{median}\left(\left|H H_{1}(i, j)\right|\right) / C, C=0.6745
$$

The results of VisuShrink are very smooth with a pleasant visual appearance. However, it is known that VisuShrink tends to over-smooth the signal, thereby losing some details (e.g. sharp edges) of the original signal that results in the increased estimation error. Because different subband and different decomposition level have different distributions, the reference[10] has usd different threshold denoising method with different decomposition level:

$$
T_{\text {new }}=\sigma \sqrt{2 \log M}\left(1+\frac{1}{j}\right)
$$

where $j=1,2, \ldots, J . J$ is the scale, i.e. the number of decomposition, the $T_{\text {new }}$ is called Multi-threshold in the paper.

\section{DENOSING WITH NEW THRESHOLD FUNCTION}

Finding the optimized threshold function is a major problem. Hard threshold function is not continuous on the threshold value $T$, so the Pseudo-Gibbs phenomena was prone to appear in the signal reconstruction. Soft threshold method processing wavelet coefficients is continuity on the whole, but there are still large deviation between the original wavelet coefficients and the estimated wavelet coefficients, it will directly affect the approximation degree between the reconstructed image and the original image. So, we try to find out optimum threshold function:

$$
\begin{gathered}
\hat{W}_{i, j}=W_{i, j} * \beta_{i, j} \\
\beta_{i, j}=\left(1-m * \frac{T_{\text {new }}^{2}}{W_{i, j}^{2}}\right)+
\end{gathered}
$$

here "+" means that the positive value should be kept as it is and the negative value should be replaced by zero. $\hat{W}_{i, j}$ is the estimation of the wavelet coefficient after using the new threshold shrinkage. The type of $m$ value is crucial, according to the reference[11,12], the estimation of noise variance can be defined as:

$$
\hat{\sigma}_{n_{i, j}}^{2}(s, J)=\frac{\operatorname{Median}\left(\left|W_{g_{i, j}}\right|(s)\right)}{C}
$$

where $W_{g_{i, j}}(1) \in\left\{L H_{J}\right\} \quad, \quad W_{g_{i, j}}(2) \in\left\{H L_{J}\right\}$, $W_{g_{i, j}}(3) \in\left\{H H_{J}\right\}, C=0.6745, s=1,2,3 ; J=1,2, \ldots l, l$ is the number of decomposition.

Because $W_{g_{i, j}}$ obeys Gauss distribution, the estimation of noisy variance can be defined as:

$$
\hat{\sigma}_{W_{g_{i, j}}}^{2}(s, J)=\frac{1}{N^{2}(J)} \sum_{i, j=1}^{N(J)} W_{g_{i, j}}^{2}(s)
$$


The value $m$ of type (7) takes for $\frac{\hat{\sigma}_{n_{i, j}}^{2}(3,2)}{\hat{\sigma}^{2}}$, using the estimation of noise variance on the $\mathrm{HH}_{2}$ subband to remove the estimation of noisy image wavelet coefficients variance on the $\mathrm{HH}_{2}$ subband, adaptive changeing the values of parameters $m$, we calculate $\beta_{i, j}$ as follows:

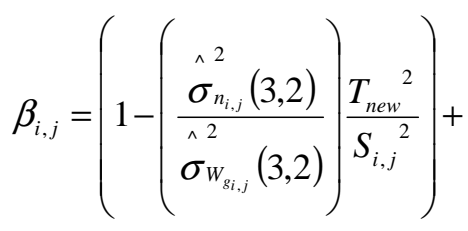

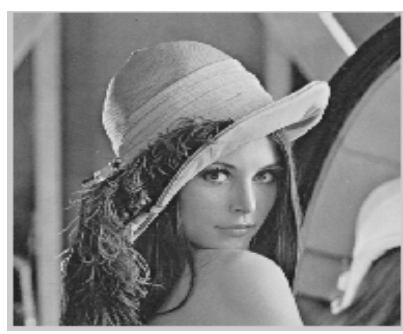

(a)

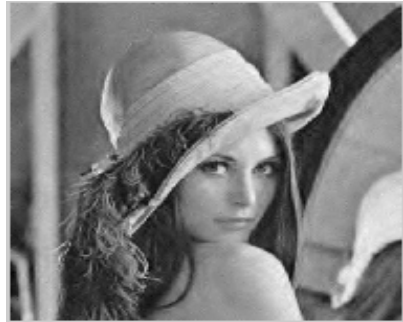

(d)

\section{EXPERIMENT RESULTS AND ANALYSIS}

Simulation experiment is carried out for in various image different levels of noise, for example, the noisy Lena images (pixel value is $512 * 512$, gray level is 256). The results of our proposed methods that use the threshold value $T_{\text {new }}$ and the new threshold function for image denoising have been compared with that of the VisuShrink soft threshold method, Multi-threshold soft threshold method and Multi-threshold hard threshold method. in order to get the better effect, not only need to select the suitable wavelet basis function, but also choose a suitable decomposition level. The high frequency information reflects the changes of image details, the edge contour caused by gray mutation , it may lead to the loss of edge information if the wavelet decomposed layer is too high. 'Db8' wavelet has the advantage of length compactly supported ,high regular, large number of vanishing moments and wavelet energy concentration. In our experiment, we have used the 'db8' wavelet and decomposition level is four .

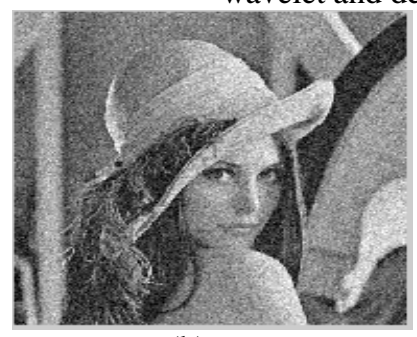

(b)

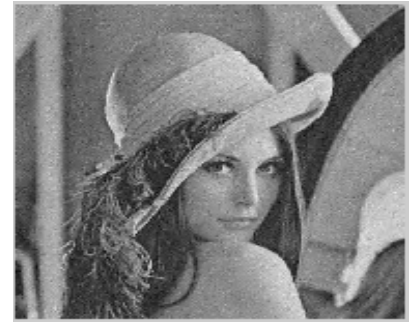

(e)

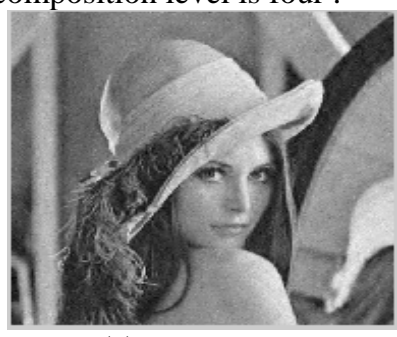

(c)

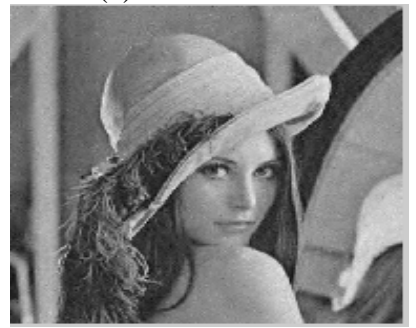

(f)

Figure 1. Lena Image:(a)Original (b)Noisy image with noise level 20 (c)Denoising using VisuShrink soft (d)Denosing using Multi-threshold soft (e)Denosing using Multi-threshold hard (f)Denosing using proposed method.

In Fig.1 (c) and Fig.1(d) the denoising image is clear, but the edge details are smoothed out and have a certain deviation with the original image. The denoising image is fuzzy and has the presence of ringing effect in Fig.1(e) . In figure 1, the denoising image Fig.1(f) is clearer and better to retain the image edge details than Fig.1 (c), Fig.1(d) and Fig.1(e) at the same time.

The purpose of this paper is to find out the estimated value of the image signal $\hat{f}(i, j)$ and the minimum mean square error ( MSE ) between $\hat{f}(i, j)$ and original image signal $f(i, j)$. In addition, we have the quantitative analysis comparison using the peak signal to noise ratio and mean square error on the denoised image. type (12) and (13) respectively mean square error(MSE) and peak signal to noise ratio(PSNR) as follows:

$$
\operatorname{MSE}(\hat{f}(i, j), f(i, j))=\frac{1}{M * N} \sum_{i, j=1}^{i=M, j=N}(\hat{f}(i, j)-f(i, j))^{2}
$$

$$
P S N R=10 \log \left(\frac{255^{2}}{M S E}\right)
$$




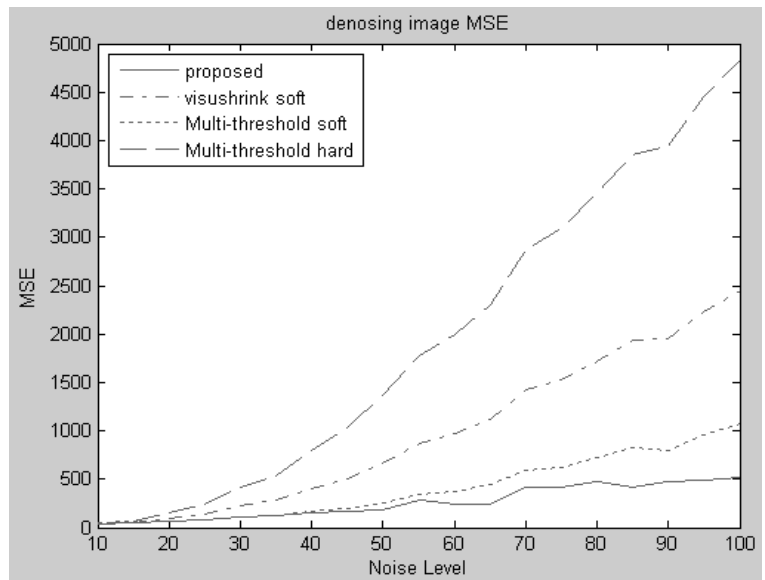

(a)

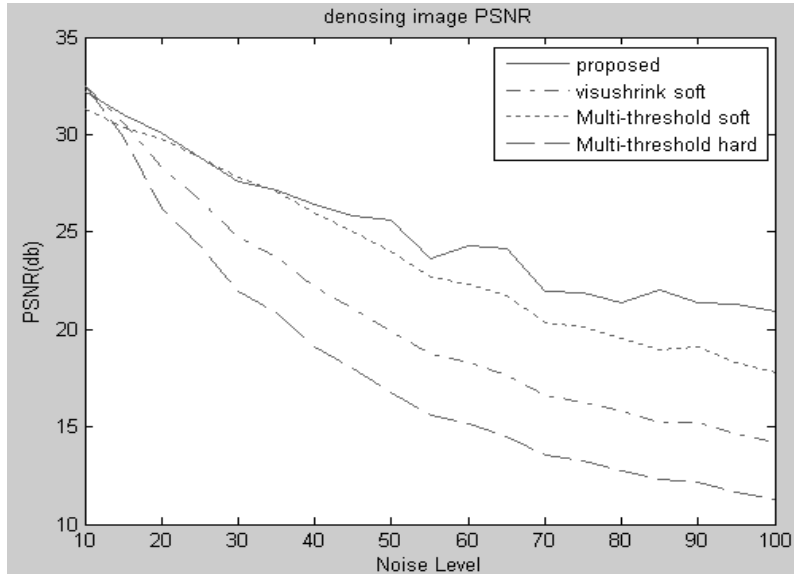

(b)

Figure 2. Lena image:(a)MSE gains, (b)PSNR gains in proposed and Visushrink soft, Multi-threshold soft,Multi-threshold hard method with noise levels of image.

In Fig.2 (a), it shows the curve changes of four different denoising methods with different noise levels under the minimum mean square error (MSE), the MSE of denoising image in proposed method is smaller than the other three methods, and the difference of MSE is bigger with the increase of noise variance. In Fig.2 (b), it shows the curve changes of four different denoising methods with different noise levels under the peak signal-to-noise ratio (PSNR), the PSNR of denoising image in proposed method is larger than the other three methods, and the difference of PSNR is bigger with the increase of noise variance. The simulation results shows that, the proposed method is superior to the traditional soft and hard threshold function, and that the effect of Multi-threshold is better than VisuShrink global threshold .

\section{CONCLUDING REMARKS}

This paper uses different thresholds for different noisy image based on the VisuShrink, and it is applied to the new threshold function. The experimental results show that the proposed threshold function can effectively remove the image noise, preserve the image edge details, obtain good denoising effect as well as denoising result in minimum mean square error ( MSE ) and peak signal to noise ratio ( PSNR ) is superior to the traditional soft and hard threshold function.

\section{ACKNOWLEDGMENT}

The authors express their sincere thanks to Prof. Quan.Haiyan for his invaluable comments and suggestions.

This work was supported by Natural Science Foundation Project of NSFC under the grant No.60872157 and No.61271007

\section{REFERENCES}

[1] Donoho.D.L,Johnstone.I.M, Ideal Spatial Adaptation via Wavelet Shrinkage[J]. Biometrika, 1994, 81:425-455.

[2] Donoho.D.L,Johnstone.I.M, Adapting to Unknown Smoothness Via Wavelet Shrinkage[J]. American Statical Assoc,1995,90(432):12001224.

[3] Donoho.D.L, Denoising By Soft threshold[J].IEEE Trans on Information Theory, 1995, 41(3): 613-627.

[4] Donoho.D.L, Wavelet Shrinkage and WVD.10-minute tour.ftp// playfair.stanford.edu.2001.

[5] L.KShark,C.Yu, Denoising by Optimal Fuzzy threshold in Wavelet Domain [J].IEEE Electronics letters,2000:36(6):581-582.

[6] Zhang.Lei,Pan.Quan, Zhang.Hongcai, Filtering Threshold Parameters in Wavelet Domain [J]. Journal of Electronics ,2001:29(3):400-402.(in chinese)

[7] S.Grace.Chang,Bin.Yu,and Martin.Vetterli, Adaptive Wavelet threshold for Image Denoising and Compression [J]. IEEE Transactions on Image Processing,2000,9:1532-1546.

[8] Hari.Om,Mantosh.Biswas, An Improved Image Denoising Method Based on Wavelet threshold[J] . Journal of Signal and Information Processing, 2012,3,109-116.

[9] Yang.Dab,Xu.Mingxing,Wu.Wenhu,et al, A Noise Cancerlation Method Based on Wavelet Transform[A].In:International Symposium on Chinese Spoken Language rocessing[C], Beijing, 2000:211-214.

[10] Feng.Xiangchu,Gan.Xiaobing,Song.Gx, Numerical Functional and Wavelet Theory [M]. Xi'an: Xi'an Electronic and Science University Press ,2003.

[11] Mantosh.Biswas* and Hari.Om, A New Soft-threshold Image Denoising Method[A]. 2nd International Conference on Communication, Computing \& Security [ICCCS-2012],Procedia Technology 6(2012) 10-15.

[12] Cha.Yufei,Bi.Duyan, Adaptive Multi Threshold Image Denoising Based on Wavelet Transform [J]. Journal of image and graphics ,Vol.10,No.5,May,2005, pp.65-69. (in chinese) 